\title{
Mechanical Model of Globular Transition in Polymers
}

\section{Citation}

Tricard, Simon, Robert F. Shepherd, Claudiu A. Stan, Phillip W. Snyder, Rebecca Cademartiri, Danny Zhu, Igor S. Aranson, Eugene I. Shakhnovich, and George M. Whitesides. 2014.

"Mechanical Model of Globular Transition in Polymers." ChemPlusChem 80 (1) (July 30): 37-41. Portico. doi:10.1002/cplu.201402203.

\section{Published Version}

doi:10.1002/cplu.201402203

\section{Permanent link}

http://nrs.harvard.edu/urn-3:HUL.InstRepos:25045868

\section{Terms of Use}

This article was downloaded from Harvard University's DASH repository, and is made available under the terms and conditions applicable to Open Access Policy Articles, as set forth at http:// nrs.harvard.edu/urn-3:HUL.InstRepos:dash.current.terms-of-use\#OAP

\section{Share Your Story}

The Harvard community has made this article openly available.

Please share how this access benefits you. Submit a story.

\section{Accessibility}




\title{
Mechanical Model of Globular Transition in Polymers
}

\author{
Simon Tricard, ${ }^{\mathrm{a}}$ Robert F. Shepherd, ${ }^{\mathrm{a}}$ Claudiu A. Stan, ${ }^{\mathrm{a}}$ Phillip W. Snyder, ${ }^{\mathrm{a}}$ \\ Rebecca Cademartiri, ${ }^{\mathrm{a}}$ Danny Zhu, ${ }^{\mathrm{a}}$ Igor S. Aranson, ${ }^{\mathrm{b}}$ Eugene I. Shakhnovich ${ }^{\mathrm{a}}$ \\ and George M. Whitesides ${ }^{\mathrm{a} *}$
}

${ }^{a}$ Department of Chemistry and Chemical Biology, Harvard University, 12 Oxford Street, Cambridge, MA 02138

${ }^{\mathrm{b}}$ Argonne National Laboratory, 9700 South Cass Avenue, Argonne, IL 60439, USA

* Author to whom correspondence should be addressed: gwhitesides@gmwgroup.harvard.edu 


\begin{abstract}
Understanding polymers in solution, in a wide range of environments - from DNA and proteins in cells to long chain polymers in gels - is important throughout science. In complex, multicomponent systems, polymers often undergo phase transitions between distinct conformations; examples include the folding of proteins, or the coil-to-globule transition of homo- and heteropolymers. This paper demonstrates a millimeter-scale granular model of coil-to-globule transitions: one "polymer" chain - a cylinders-on-a-string "pearl necklace" - and many spheres, all shaken on a horizontal surface. This model includes short- and long-range interactions and is more complex than most granular models of molecular systems. It is possible to describe the behavior of this granular system using formalisms generally used in statistical physics of polymers, i.e. first and second order coil-to-globule transitions. This designed granular system represents another kind of approach to the study of polymer phase transitions and might inspire future designs of polymer-like mesoscale systems.
\end{abstract}




\section{Main text}

\section{Introduction}

Although a homo-polymeric molecule experiencing Brownian motion in solution, and a "pearl necklace" shaken on a horizontal flat surface in an assembly of spheres, are very different systems, they exhibit surprisingly similar statistical-physical behaviors. The problem we have explored is the extent to which systems topologically analogous to polymers, but with a scale of components large enough to be visible and with numbers of components small enough that all can be accounted for explicitly, exhibit complex phenomena such as phase transitions. Such systems could provide a tool for improving intuition about molecular phase transitions, and guide the design of molecular and nanoscale systems that exhibit such transitions.

The folding of proteins to the native state, or the coil-to-globule transition of homo- and hetero-polymers are examples of complex behaviors of long chain molecules in solution. ${ }^{1-4}$ Numerous theoretical and experimental studies are actively performed to understand these transitions, and often involve intensive computations, or sophisticated experimental methods. ${ }^{5-9}$ In parallel, solid-liquid granular phase transitions have been reported, and described in detail for layers on horizontally vibrating devices. ${ }^{10-12}$ For example, Clerc et. al. reported a granular solidliquid phase transition in a vibrating granular monolayer in one dimension, ${ }^{13}$ and Castillo et. al. extended this description to two dimensions. ${ }^{14}$

Here we designed a granular system to simulate a phase transition that is ubiquitous in homo-polymers in solution: the transition from an expanded fluctuating coil state to a collapsed globule state, which depends on the intermolecular interactions between the polymer and the surrounding solvent, and on intramolecular interactions between different regions of the polymer. ${ }^{1,15}$ If the solvent is a "good" one, the polymer chain will expand as the temperature of 
the system increases; if it is a "poor" solvent, the chain will collapse. This transition is usually second-order (continuous), but there is evidence that it could occur as a first-order (discontinuous) transition as well. , $^{56}$

We previously introduced "mechanical agitation" (MecAgit) as a method for physical simulation of the behavior of microscopic systems, using a two-dimensional arrangement of millimeter-sized components that move under a pseudo-random agitation. ${ }^{17,18}$ Even though MecAgit systems are macroscopic, out-of-equilibrium, and dissipative, and even though their motions are not completely random, we used them to mimic experimentally the folding of a short chain RNA, ${ }^{19}$ the statistics of conformations of worm-like chain (WLC) polymers, ${ }^{17}$ and the development of a Boltzmann distribution. ${ }^{18}$ Other groups have used chain models as well: BenNaim et. al. studied topological constraints such as knots, ${ }^{20}$ Safford et. al. the effect of confinement, ${ }^{21}$ Zou et. al. glass transition in polymers, ${ }^{22}$ Blair et. al. the formation of chains by shaking magnetic beads and free particles, ${ }^{23}$ and Yuan et.al. segregation in mixtures of granular chains and spherical grains. ${ }^{24}$ The novelty of our approach is the focus on designing granular models of phase transitions in polymer physics; our models have multiple phenomenological similarities to the behavior of polymers. We achieved this purpose by engineering inter-particle interactions and tailoring mechanical agitation.

Here, we use polymer statistical physics to describe the collapse of macroscopic cylinders-on-a-string shaken in an assembly of free spheres. We shook millimeter-sized cylinders-on-a-string (the "polymer chain") on a flat horizontal surface within a region bounded by vertical walls, in the presence of a variable number of free spheres (Fig. 1a). We changed the filling ratio $(F R)$ of free spheres: that is, the ratio between the number of spheres in the system and the number of spheres required to completely fill the available area with a single layer of spheres having compact hexagonal packing (for a closed-packed packing, $F R=100 \%$ ). Unlike 
other horizontally shaken granular systems, ${ }^{12,25}$ ours has a pseudo-random agitation motion, which results from a combination of an orbital shaking of the surface, and randomization by agitation of a pendulum hanging underneath, with a linear actuator. ${ }^{19}$ This type of agitation randomizes some aspects of the motion of the chain (although the motion of the spheres is strongly correlated) such that this motion could be described to a useful approximation by a "macroscopic temperature": that is, a parameter that had a simple experimental relationship to the frequency of the orbital shaker, $f$, and described the steady-state behavior of the chain. ${ }^{17,18}$ This granular system is a model of polymers in solution: the cylinders-on-a-string represent a polymer molecule, and the assembly of free spheres a thermal bath of solvent molecules. To characterize the folding state of the chain we used its radius of gyration, $R_{g}$, defined as the root-mean-square distance between the monomer units and the center of mass of the polymer: ${ }^{26}$

$$
R_{g}=\sqrt{\frac{1}{N} \sum_{k=1}^{N}\left(r_{k}-r_{C M}\right)^{2}}
$$

where $N$ is the number of cylinders, $r_{k}$ are the positions of the cylinders and $r_{C M}$ is the position of the center of mass of the chain.

\section{Results}

Modeling the second-order coil-to-globule phase transition

First, we studied a chain made of ten cylinders threaded on a string and separated by small spheres that allowed the chain to bend (Fig. 1b). ${ }^{19,27}$ The contour length of the chain was equal to $23.9 \mathrm{~cm}$; in absence of free spheres, the chain had an extended (but not completely stretched) conformation under agitation that was described by a worm-like chain (WLC) behavior with a persistence length equal to $12.2 \mathrm{~cm} .{ }^{17}$ Under certain experimental conditions, the chains collapsed after collisions with free spheres (Fig. 1c). In a three-bead chain, the collapse, as we described 
previously, ${ }^{18}$ is a consequence of imbalanced rates of collision with spheres between the open and the closed sides of the chain - the spheres exert a "pressure" that can close the chain if it overcomes its intrinsic rigidity. Extrapolating this mechanism, the collapse of longer chains will occur if the "sphere pressure", which increases at larger filling ratios, is strong enough. ${ }^{25}$ The collapsed conformation was not static, though: the chain reached a steady state in which it continuously stretched and shrunk, but never returned to a completely extended conformation (Fig. 1d).

Once the chain reached the steady state, we took multiple snapshots of the system at equally-spaced time intervals to determine the more likely conformations and their probability of occurrence. The steady-state distribution of $R_{g}$ is shown as a function of $f$ and $F R$ in Fig. 2a to 2e. At a low filling ratio of free spheres $(F R=40 \%)$, the chain always stayed in an extended (but not stretched) conformation: the filling ratio of free spheres was sufficiently low that it did not perturb the behavior the chain displayed in their absence. When we increased $f$ at $\mathrm{FR}=40 \%$, the distribution of $R_{g}$ broadened and shifted continuously towards lower values - a WLC behavior that we also observed in an analogous system without spheres. ${ }^{17}$ From $F R=50 \%$ to $F R=70 \%$, the mean value of the $R_{g}$ distribution, denoted as $\left\langle R_{g}\right\rangle$, first increased with $f$, and then decreased, as we varied $f$ from low to high values (Fig. $2 \mathrm{f}$ ). Since, in our system, $f$ plays the role of temperature, the change in the sign with which the system responded to variations in $f$ indicates that the chain transitioned between two states with different "temperature" dependencies. In analogy with the coil and globule states of polymers, in which the radius of gyration has different temperature dependencies, we identified a coiled state that corresponded to a chain with $\left\langle R_{g}>\right.$ increasing with $f$, and a globular state that corresponded to a chain with $<R_{g}>$ decreasing with $f$. We calculated the agitation frequency at which the transition occurred, $f_{0}(F R)$, as the intersection of the linear regressions corresponding to each behavior (Fig. $2 \mathrm{f}$ ): $f_{0}$ 
$(F R=50 \%)=99 \mathrm{rpm}, f_{0}(F R=60 \%)=97 \mathrm{rpm}$, and $f_{0}(F R=70 \%)=88 \mathrm{rpm} ; f_{0}$ is the extrapolated frequency at which $<R_{g}>$ is maximum. When a large fraction of free spheres was present on the dish $(F R=80 \%)$, the chain remained collapsed throughout agitation. The distribution of $R_{g}$ was narrow and its average $<R_{g}>$ independent of $f$. This behavior corresponds to a compact globule state of a polymer molecule. ${ }^{1}$

Fig. 2g summarizes the previous descriptions as a four-phase (WLC-type, coil, globule, and compact globule) diagram in the $(f, F R)$ space. We performed Kolmogorov-Smirnov tests on $R_{g}$ distributions on either side of the frontiers between the phases, to confirm they were statistically distinct: between the worm-like chain and the coil-globule phases (same $f, F R=40 \%$ and $F R=50 \%$ ), between the coil-globule and the compact globule phases (same $f, F R=70 \%$ and $F R=80 \%)$, and between the coil and the globule phases ( $f$ below and above $f_{0}$, same $F R$ ). The pvalues for all the tests were lower than 0.03 . The transitions between the phases of the diagram were thus quantitative at the scale of the experimental meshing of the $(f, F R)$ space.

In addition to its dependence on $f$, the unimodality of the $R_{g}$ distribution for all the $(f, F R)$ conditions between $F R=50 \%$ and $F R=70 \%$, is another signature of a second-order transition. ${ }^{1}$ The coil-to-globule transition of MecAgit model described here is thus analogous to a coil-toglobule transition in polymers, induced by a change in temperature. The assembly of spheres is analogous to a "poor" solvent with a lower critical solution temperature (LCST), as observed for some polymers and proteins. ${ }^{6-8}$ When $f$ increased, the model chain exhibited the gradual compaction characteristic of second-order phase transitions of polymer chains when the temperature rises above the $\theta$-point (i.e., where the second virial coefficient changes sign from positive to negative)..$^{9,26,28}$ 


\section{Modeling the first-order coil-to-globule phase transition}

First-order phase transitions are encountered in some polymer systems - such as charged polymers - but experimental proofs of such transition at the molecular scale remains controversial, and their mechanistic origin is still a subject of investigation. ${ }^{1,5,16}$ To observe such transitions in our granular system, we designed a system where two antagonistic forces acted at different scales: we chose a thicker thread that stabilized the extended conformation of the chain, and we inserted magnetic cylinders (Fig. 3a) to add attractive interactions between the plastic cylinders of the chain and favor the collapsed conformation. The length scale of the elastic interaction due to the stiffness of the thick thread is on the order of magnitude of the persistence length of the chain - i.e. tens of centimeters - while the length scale of the attraction between the magnets is a few centimeters.

Under agitation, the chain had two primary conformations: one ring-shaped, and one extended (Fig. 3b). The ring-shaped conformation was the more compact conformation; the rigidity of the thread prevented compaction below the ring size for the extended chain. The existence of two primary conformations was confirmed by two distinct peaks in the distributions of $R_{g}$ : one centered at $4.0 \mathrm{~cm}$, which corresponded to the ring-shaped conformation, and one centered at $7.2 \mathrm{~cm}$, which corresponded to the extended conformation (Fig. 3c). At high and low coverage of the surface by free spheres, the distributions of $R_{g} \operatorname{did}$ not depend on $f$ : For $F R \geq$ $90 \%$, the chain collapsed to the ring-shaped conformation, and for $F R \leq 60 \%$, the chain remained in the extended conformation. For intermediate $F R$, we observed both distributions, and the predominant conformation evolved from extended to ring-shaped as $f$ was increased. For the $(f$, $F R$ ) conditions where the two distributions were present, the chain opened and closed from the extended to the ring-shaped conformation randomly. The evolution of $\left\langle R_{g}>\right.$ as a function of $f$ 
could be fitted approximately by a sigmoid, where $f_{0}$ was the frequency at the center of the sigmoid and depended on $F R: f_{0}(F R=60 \%)=284 \mathrm{rpm}, f_{0}(F R=70 \%)=112 \mathrm{rpm}, f_{0}(F R=80 \%)$ $=101 \mathrm{rpm}, f_{0}(F R=90 \%)=64 \mathrm{rpm}$ (Fig. 3d). Fig. 3e summarizes the previous discussions in a phase diagram; the $f_{0}$ values correspond to the frequencies of transition.

At low $F R$, the tension of the string is predominant (high persistence length) and the magnetic attractions are negligible. When we increased FR, the "pressure" of the free spheres counteracted the tension of the string, making it more compact; in this conformation the shortranged magnetic attractions began to be significant. Both the "pressure" of the free sphere and the attraction of the magnetic beads favored the collapsed state. The presence of a bimodal $R_{g}$ distribution, which correspond to two distinct conformations with peaks at specific $f$ values, and the sigmoid shape of the evolution of $\left\langle R_{g}>\right.$ as a function of $f$ are typical signatures of a firstorder transition.

\section{Discussion}

The collapse of the chain in our granular system is favored by the phase separation between the cylinders of the chain and the free rolling spheres. These two types of components react differently to the external conditions: they have different responses to friction, and different responses to agitation (due to their different shapes, masses and inertias). When a component is surrounded by homologous ones, all the particles experience the same driving force and only few interparticle collisions occur. When two components are different, they feel different driving forces and experience frequent collisions. The distinct mobility of different types leads to their separation, which tends to reduce the rate of collisions as much as possible. ${ }^{29,30}$ Phase separation is enhanced when the density of the components is increased and when external driving forces are increased; ${ }^{12}$ that is why the collapsed conformations of the chains were favored at high $F R$ and 
high $f$. Increasing both $F R$ and $f$ corresponds to increasing both the density and the velocity of spheres around a given component of the other type, and thus to larger net pressures on it. ${ }^{25}$

Although the nature of the strongest forces acting on particles is different at the mesoscopic granular scale (friction, hard-sphere collisions, and here magnetic dipole forces) than at the microscopic scale (electrostatic and Van der Waals forces), we observed that cylinders-ona-string demonstrated statistical behaviors analogous to those of molecular homo-polymers in solution. The physical simulations reported here go beyond a simple topological analogy, as we introduced intra-chain forces at different length scales and with different signs to generate a type of folding transition that is uncommon in polymers and not well understood. As an example of coil-to-globule transitions, our work may be relevant to protein folding and to genome folding., ${ }^{4,31}$

First-order transitions, as for example liquid-vapor transitions, occur classically between a low-energy, low-entropy state (liquid) and a high-energy, high-entropy state (vapor). Here, we are dealing with an out-of-equilibrium system, and thermodynamic arguments have limited relevance. Moreover, both states in the present system have low entropy as they represent single conformations. Nonetheless, introducing interactions at two different scales gave rise to only two observable conformations; intermediate conformations were not apparent in the statistical description of the system, i.e. the $R_{g}$ distributions. In some sets of $(f, F R)$ conditions $-e . g$. for $(f$, $F R)=(110 \mathrm{rpm}, 70 \%),(120 \mathrm{rpm}, 70 \%),(100 \mathrm{rpm}, 80 \%),(110 \mathrm{rpm}, 80 \%)-$ the $R_{g}$ distribution had two distinct peaks, corresponding to the ring-shaped and extended conformations. Thus the system was not a simple switch (for which we would have observed only one peak in the distribution for a given set of $(f, F R)$ conditions $)$ but evolved as in a first-order transition scenario. The distributions of $R_{g}$ around the two observable conformations related to two distinct ensembles of accessible states, characterized by different "state densities" (corresponding to different arrangements of the chain). The system could thus perform a discontinuous transition 
between two ensembles of states, and states with intermediate densities were possible but unstable, and not observed statistically - like in first-order phase transitions.

The MecAgit approach is novel for three reasons: 1) We used a more complex system than all the previously reported ones, with the introduction of two antagonistic forces at different scales, which led to a first order phase transition; to our knowledge, this effect has never been reported before with granular models (most of them were simply vertically vibrated, leading to a fundamentally different behavior). 2) The origin of a first-order phase transition is still a subject of debate in the polymer physics community; we proved, by a physical experiment, that the hypothesis of using two antagonistic forces at different scales is a valid hypothesis. 3) Our work demonstrates that phenomenological analogies are important and gives insights into complex behaviors. While the precise mechanistic details are different between granular systems and molecules in solution, we were able to validate a general principle that can determine the order of phase transitions in microscopic systems.

While our ability to change the order of the folding transition by adding competing longand short- range interactions might suggest that a similar approach would work at the molecular scale, its success is not certain. Controlling interactions at a molecular level is a real challenge, as numerous inter-dependant experimental conditions are present simultaneously: temperature, nature of the monomers, length and rigidity of the polymer, nature of the solvent, concentration of the solutes, etc. Polymer-like mesoscale systems reported by others, however, might provide better opportunities for designing phase behavior. For example, Cademartiri et. al. have shown recently that $\mathrm{Bi}_{2} \mathrm{~S}_{3}$ nano-wires presented polymer-like conformation and growth kinetics, and Liu et. al. demonstrated the possibility to direct the self-assembly of gold nano-rods in linear conformations, just as monomers assemble to form polymers. ${ }^{32}$ Our work, which shows that the engineering of inter-particle interactions is a valid approach to control phase transitions in 
millimeter-scale granular systems, might thus inspire the design of future polymer-like mesoscale systems.

\section{Methods}

Summary. We prepared a circular mixing area with a diameter of $0.48 \mathrm{~m}$ using an aluminum rim, and we covered the area inside the rim with paper to generate an area with a constant friction coefficient on which the objects would roll, but not slide, when the plate was agitated. To avoid any possible electrical charging by contact electrification within the experimental setup, we maintained a relative humidity of more than $60 \% \mathrm{RH}$ using a humidifier connected to the enclosed space above the plate. ${ }^{19}$ In all experiments, we filled the mixing area with simple (spheres) and composite (cylinders connected by a string) polymeric objects. We varied the filling ratio $(F R)$ of spheres between $40 \%$ and $90 \%$ (see the definition of $F R$ in the main text). To simulate molecular phenomena in the presence of thermal agitation, we shook the system with a combination of orbital translation (with frequencies $f$ from $80 \mathrm{rpm}$ to $150 \mathrm{rpm}$ ) and randomly timed flicks to make the movement of particles aperiodic. The movement of the cylinders-on-astring was followed by taking snapshots with a picture camera. For each $(f, F R)$ condition, we took one hundred pictures at regular intervals to probe all the possible conformation taken by the chain. We extracted the $R_{g}$ values for each picture with a custom-made program.

Materials. Nylon-6,6 rods with a diameter of $6 \mathrm{~mm}$ and acrylic spheres with a diameter of $3 \mathrm{~mm}$ were purchased from McMaster-Carr Supply Company. We cut the rods into $14 \mathrm{~mm}$-long cylinders. Holes with a diameter of $1.3 \mathrm{~mm}$ were drilled through the spheres and the cylinders. 6$\mathrm{mm}$ wide transparent acrylic spheres were purchased from Engineering Laboratories. The 
aluminum honeycomb panel was purchased from Home Depot. Silver-coated, $2 \times 2$-mm crimp beads were purchased from Beadalon. Nylon-6,6 threads with a diameter of $75-\mu \mathrm{m}$ and $600-\mu \mathrm{m}$ were purchased from thethreadexchange.com. Fadeless ${ }^{\circledR}$ paper was purchased from Pacon Corporation. Magnetic hematite $3 \times 9$-mm tubes were purchased from Arizona Bead Company. To stain the transparent nylon cylinders blue, we immersed them in an aqueous solution of Disperse Blue 14 and placed on a hot plate at a temperature of $100{ }^{\circ} \mathrm{C}$ for $30 \mathrm{~min}$. Dyed cylinders were washed with water and ethanol and dried with a $\mathrm{N}_{2}$ stream.

Construction and Agitation of a Large Flat Surface. The flat surface on which the objects were agitated $(1.2 \times 1.2 \mathrm{~m}$ square, $\sim 7 \mathrm{~kg}$ weight $)$ was made from an aluminum honeycomb panel and was supported from the ceiling via an elastic cord joined to four cables which were anchored to the corners of the panel. The distance between the plate and the attachment point to the ceiling was $1.3 \mathrm{~m}$. We attached to the center of the plate, below it, a pendulum made from a light $(\sim 0.1$ $\mathrm{kg}$ ) $0.3-\mathrm{m}$ rigid rod with an end weight with a mass of $1.4 \mathrm{~kg}$. The pyramid defined by the panel and the cables was wrapped in a polyvinyl chloride (PVC) film to control the humidity above the plate. ${ }^{19}$ We maintained a relative humidity higher than $60 \%$ using an air humidifier (Vicks V5100NS). To record snapshots of the system during agitation, we used a photo camera (Nikon D40) that was suspended $\sim 1 \mathrm{~m}$ vertically above the plate. To achieve pseudo-random agitation, we applied two shaking drives: one from an orbital shaker attached to the plate, and one from a linear actuator which kicked the weight of the pendulum under the plate. The orbital shaker (Madell Technology Corp., ZD-9556-A) was attached to the plate via an elastic polyurethane cord, and the motion it imparted to the plate was a combination of orbital translation with a small-amplitude angular oscillation. The radial amplitude of the orbital shaker was $5.1 \mathrm{~mm}$, and its frequency was variable; we used orbital frequencies ranging from $f=80$ to $f=150$ revolutions 
per minute (rpm). The second shaking motion had the role of randomizing the orbital motion. We kicked with a linear actuator (LinMot, Inc., P01-23x80) the weight under the plate; the linear actuator moved at a fixed frequency of $4 \mathrm{~Hz}$ in all experiments, but the times at which it impacted the pendulum were not periodic due to the complicated motion of the pendulum. The frequency of kicks was higher than the frequency of orbital shaking, and the system did not complete a full orbit between kicks to the pendulum. The overall motion of the plate was aperiodic but not entirely random, and we estimated that the maximum horizontal acceleration of the plate was approximately $5 \mathrm{~m} / \mathrm{s}^{2}$. The area of agitation of the components (spheres and cylinders-on-astring) was defined by an aluminum rim with a diameter of $0.48 \mathrm{~m}$. We covered the surface of the board with paper to control the movement of the spheres and the cylinders; the roughness and softness of the paper was such that the objects consistently rolled but did not slide.

Preparation of the Cylinders-on-a-string. The flexible links in the chain were weakly elastic and were made from a nylon string on which we strung three 3.18-mm diameter PMMA spheres. Aluminum crimps fixed the cylinders on the string and controlled the elasticity of the chain, as described previously. ${ }^{17-19}$ We chose a system of ten cylinders with a contour length $L=23.9 \mathrm{~cm}$. For the non-magnetic chain, we used a Nylon-6,6 threads with a diameter of $75 \mu \mathrm{m}$ and Nylon cylinders. The persistence length was previously determined and was equal to $L_{p}=12.2 \mathrm{~cm} .^{17}$ When we used several chains, the system jammed in few minutes - because of a high number of inelastic collisions, which are known to lead to clustering. ${ }^{33}$ For the magnetic chain, we kept the same sequence, but we chose a Nylon-6,6 thread with a diameter of $600 \mu \mathrm{m}$ and magnetic cylinders. To fabricate the magnetic cylinders, we enlarged the holes of the Nylon cylinders to a diameter of $3.2 \mathrm{~mm}$ and glued hematite tubes inside with epoxy glue. 
Image Analysis. Two different designs of colors have been used. For the study with the nonmagnetic cylinders, we used a white paper background and gray cylinders-on-a-string; we stained cylinders at the termini to increase the visual contrast between the middle cylinders of the chain and the cylinders at the ends of the chain and to facilitate image analysis. For the study with the magnetic cylinders we dyed all the cylinders in blue and used a brown paper background; this choice of colors improved the reliability of the image analysis. The free acrylic spheres are transparent in both cases but appeared white on the images because of the reflection of the light from the flash of the camera. We observed the evolution in time of the radius of gyration of the chain by collecting one picture of the system under continuous agitation. We adapted the time of measurements to the frequency of agitation, so that the chain probed all the possible conformations: every $1 \mathrm{~min}$ for $f=80 \mathrm{rpm}$ to $100 \mathrm{rpm}$, every $30 \mathrm{~s}$ for $f=110 \mathrm{rpm}$ to $130 \mathrm{rpm}$, and every $20 \mathrm{~s}$ for $f=140 \mathrm{rpm}$ and $150 \mathrm{rpm}$. All experiments were started in an extended conformation, but we waited until the system reached a steady state before recording images. We collected data only when the system could freely evolve and explore all the possible conformations, i.e. when the system was not jammed because of a too high $F R$ or a too low $f$. The radius of gyration was calculated averaging results form 100 pictures. The radius of gyration of the chain was determined with custom-made script developed in Java that analyzed each frame separately. The script used a sequence of threshold operations, erosions, dilations, and removal of small-area signals to produce monochrome images that preserved only the image of the cylinders; from such images, the position of the end cylinders and the center-of-mass of the chain can be computed automatically. The details of the operations in the sequence were optimized separately for the two types of chains used (magnetic and non-magnetic), resulting in two distinct imageprocessing procedures. In both cases, since the processes were not entirely reliable, each processed image was shown to a human viewer, with the detected cylinder center locations 
marked on the image. The viewer could then edit the locations if they were incorrect. This system allowed the cylinders to be detected more accurately and quickly, and with less work, than a purely manual system, while maintaining high accuracy. After the verification of the processed images, we calculated the radius of gyration from the coordinates of the centers of mass of the cylinders for each frame in the image sequence.

\section{References}

1. Grosberg, A. Y. \& Khohlov, A. R. Statistical Physics of Macromolecules. (American Institute of Physics, 1994).

2. Quake, S. R., Babcock, H. \& Chu, S. The dynamics of partially extended single molecules of DNA. Nature 388, 151-154 (1997).

3. Fredrickson, G. H., Ganesan, V. \& Drolet, F. Field-theoretic computer simulation methods for polymers and complex fluids. Macromolecules 35, 16-39 (2002).

4. Shakhnovich, E. Protein folding thermodynamics and dynamics: Where physics, chemistry, and biology meet. Chem. Rev. 106, 1559-1588 (2006).

5. Yu, J. Q., Wang, Z. L. \& Chu, B. Kinetic-Study of Coil-to-Globule Transition. Macromolecules 25, 1618-1620 (1992).

6. Montesi, A., Pasquali, M. \& MacKintosh, F. C. Collapse of a semiflexible polymer in poor solvent. Phys. Rev. E 69, 021916 (2004).

7. Lucent, D., Vishal, V. \& Pande, V. S. Protein folding under confinement: A role for solvent. Proc. Nat. Acad. Sci. 104, 10430-10434 (2007).

8. Grater, F., Heider, P., Zangi, R. \& Berne, B. J. Dissecting entropic coiling and poor solvent effects in protein collapse. J. Am. Chem. Soc. 130, 11578-11579 (2008).

9. Simmons, D. S. \& Sanchez, I. C. A model for a thermally induced polymer coil-to-globule transition. Macromolecules 41, 5885-5889 (2008).

10. Olafsen, J. S. \& Urbach, J. S. Two-dimensional melting far from equilibrium in a granular monolayer. Phys. Rev. Lett. 95 (2005).

11. Aranson, I. S. \& Tsimring, L. S. Patterns and collective behavior in granular media: Theoretical concepts. Rev. Mod. Phys. 78, 641-692 (2006).

12. Aranson, I. S. \& Tsimring, L. S. Granular patterns. (Oxford University Press, 2009). 
13. Clerc, M. G. et al. Liquid-solid-like transition in quasi-one-dimensional driven granular media. Nat. Phys. 4, 249-254 (2008).

14. Castillo, G., Mujica, N. \& Soto, R. Fluctuations and Criticality of a Granular Solid-Liquid-Like Phase Transition. Phys. Rev. Lett. 109 (2012).

15. Dill, K. A. \& Bromberg, S. Molecular Driving Forces. (Garland Science, 2011).

16. Maffi, C., Baiesi, M., Casetti, L., Piazza, F. \& De Los Rios, P. First-order coil-globule transition driven by vibrational entropy. Nature Communications 3 (2012).

17. Tricard, S. et al. Analog modeling of Worm-Like Chain molecules using macroscopic beads-on-astring. Phys. Chem. Chem. Phys. 14, 9041-9046 (2012).

18. Tricard, S., Stan, C. A., Shakhnovich, E. I. \& Whitesides, G. M. A macroscopic device described by a Boltzmann-like distribution. Soft Matter 9, 4480-4488 (2013).

19. Reches, M., Snyder, P. W. \& Whitesides, G. M. Folding of electrostatically charged beads-on-astring as an experimental realization of a theoretical model in polymer science. Proc. Nat. Acad. Sci. 106, 17644-17649 (2009).

20. Ben-Naim, E., Daya, Z. A., Vorobieff, P. \& Ecke, R. E. Knots and random walks in vibrated granular chains. Phys. Rev. Lett. 86, 1414-1417 (2001).

21. Safford, K., Kantor, Y., Kardar, M. \& Kudrolli, A. Structure and dynamics of vibrated granular chains: Comparison to equilibrium polymers. Phys. Rev. E 79, 061304 (2009).

22. Zou, L. N., Cheng, X., Rivers, M. L., Jaeger, H. M. \& Nagel, S. R. The Packing of Granular Polymer Chains. Science 326, 408-410 (2009).

23. Blair, D. L. \& Kudrolli, A. Clustering transitions in vibrofluidized magnetized granular materials. Phys. Rev. E 67, 021302 (2003).

24. Yuan, X. X., Zheng, N., Shi, Q. F., Sun, G. \& Li, L. S. Segregation in mixtures of granular chains and spherical grains under vertical vibration. Phys. Rev. E 87 (2013).

25. Aumaitre, S., Kruelle, C. A. \& Rehberg, I. Segregation in granular matter under horizontal swirling excitation. Phys. Rev. E 64, 041305 (2001).

26. Rubinstein, M. \& Colby, R. C. Polymer physics. (Oxford University Press, 2003).

27. Even if a chain of ten cylinders is strictly speaking an "oligomer", we keep the generic "polymer" term to stay general.

28. Lifshitz, I. M., Grosberg, A. Y. \& Khokhlov, A. R. Some Problems of Statistical Physics of Polymer-Chains with Volume Interaction. Rev. Mod. Phys. 50, 683-713 (1978).

29. McCandlish, S. R., Baskaran, A. \& Hagan, M. F. Spontaneous segregation of self-propelled particles with different motilities. Soft Matter 8, 2527-2534 (2012).

30. Farrell, F. D. C., Marchetti, M. C., Marenduzzo, D. \& Tailleur, J. Pattern Formation in SelfPropelled Particles with Density-Dependent Motility. Phys. Rev. Lett. 108 (2012). 
31. Lieberman-Aiden, E. et al. Comprehensive Mapping of Long-Range Interactions Reveals Folding Principles of the Human Genome. Science 326, 289-293 (2009).

32. Liu, K. et al. Step-Growth Polymerization of Inorganic Nanoparticles. Science 329, 197-200 (2010).

33. Goldhirsch, I. \& Zanetti, G. Clustering Instability in Dissipative Gases. Phys. Rev. Lett. 70, 1619$1622(1993)$.

Figure 1. Qualitative behavior of a macroscopic chain made of plastic cylinders agitated in a bath of free spheres. (a) Experimental apparatus. Polymer spheres and a chain with flexible links are shaken with an aperiodic motion on a horizontal surface. (b) Schematic of the flexible chain. Ten Nylon cylinders (white and blue) were threaded on a Nylon string and separated by smaller acrylic spheres (gray) that tuned the flexibility of the cylinders-on-a-string. To facilitate image analysis, we dyed the cylinders at the termini of the chain blue. (c) Time-lapse photography (every $30 \mathrm{~s}$ ) of the initial collapse of the chain, starting from an extended conformation. (d) Time-lapse photography (every 2 minutes) of the steady behavior of the chain. The collapse was dynamic, with the chain continuously elongating and folding. In (b) and (c), the filing ratio of free spheres $F R$ was fixed at $80 \%$ and the frequency of the orbital shaker $f$ was set at $130 \mathrm{rpm}$. Scale bars: $2 \mathrm{~cm}$. 
Figure 2. Physical model of a second-order coil-to-globule transition. We agitated a chain made of ten cylinders in a bath of free spheres (same chain as in Figure 1). (a) to (e), Histograms of the evolution of the distribution of the radii of gyration $\left(R_{g}\right)$ of the chain, at different frequencies of the orbital shaker $f(\mathrm{rpm})$, when the filling ratios of free spheres $F R$ was increased: $F R=40 \%(\mathrm{a}), 50 \%(\mathrm{~b}), 60 \%(\mathrm{c}), 70 \%(\mathrm{~d}), 80 \%(\mathrm{e})$. In graphs (b) to (d), the plots at low $f$ values (I) were separated from plots at high $f$ values (II) for clarity; the average value of $R_{g}$ increased with $f$ in I, whereas it decreased in II. The lines connect experimental data. (f) Evolution of the average radii of gyration $\left\langle R_{g}>\right.$, and their linear regressions, as a function of $f$ for different $F R$. The error bars represent 95\% confidence intervals. (d) Phase diagram of the experimental model system as a function of $f$ and $F R$. The error bars represent the spread of frequency values in the area of overlap between $68.3 \%$ confidence intervals of the two linear fits whose intersection defines the phase transition frequencies $f_{0}$.

Figure 3. Physical model of a first-order coil-to-globule transition. We agitated a chain with a high persistence length made of ten magnetic cylinders in a bath of free spheres. (a) Schematic of the cross-section of an individual cylinder. (b) Representative photographs of the two equilibrium conformations of the chain: extended (left) and ring-shaped (right). Scale bar: $2 \mathrm{~cm}$. (c) Histograms of the evolution of the distribution of radii of gyration $R_{g}$ of the chain, at different frequencies of the orbital shaker $f$, when the filling ratio of free spheres $F R$ was increased. The lines connect experimental data. (d) Evolution of the average radii of gyration $\left\langle R_{g}>\right.$, and their sigmoidal fits, as a function of $f$ for different $F R$. The error bars represent $95 \%$ confidence intervals. (e) Phase diagram of the experimental model system as a function of $f$ and $F R$. The error bars correspond to the $95 \%$ confidence intervals of the sigmoidal fits in Fig. 3(d). In both 
Fig 3(d) and 3(e), we only indicate the direction where $f_{0}(60 \%)$ and $f_{0}(90 \%)$ are located, as they are outside the range of frequencies investigated experimentally.

\section{Acknowledgements}

This work was supported by the US Department of Energy, Division of Materials Sciences \& Engineering, under Award No. DE-FG02-00ER45852. Research of IS Aranson was supported by the U.S. DOE, Office of Basic Energy Sciences, Division of Materials Science and Engineering, under Contract No. DE AC02-06CH11357.

\section{Author contributions}

ST, RFS, PWS and EIS designed the experiments. ST, RFS, RC and DZ performed the experiments. ST, CAS, ISA, EIS and GMW interpreted data. DZ wrote the image analysis program. ST, CAS and GMW wrote the manuscript. All authors discussed the results and implications and commented on the manuscript at all stages.

\section{Competing financial interests}

The authors declare no competing financial interests. 

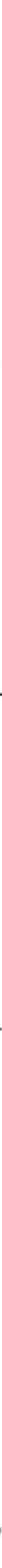

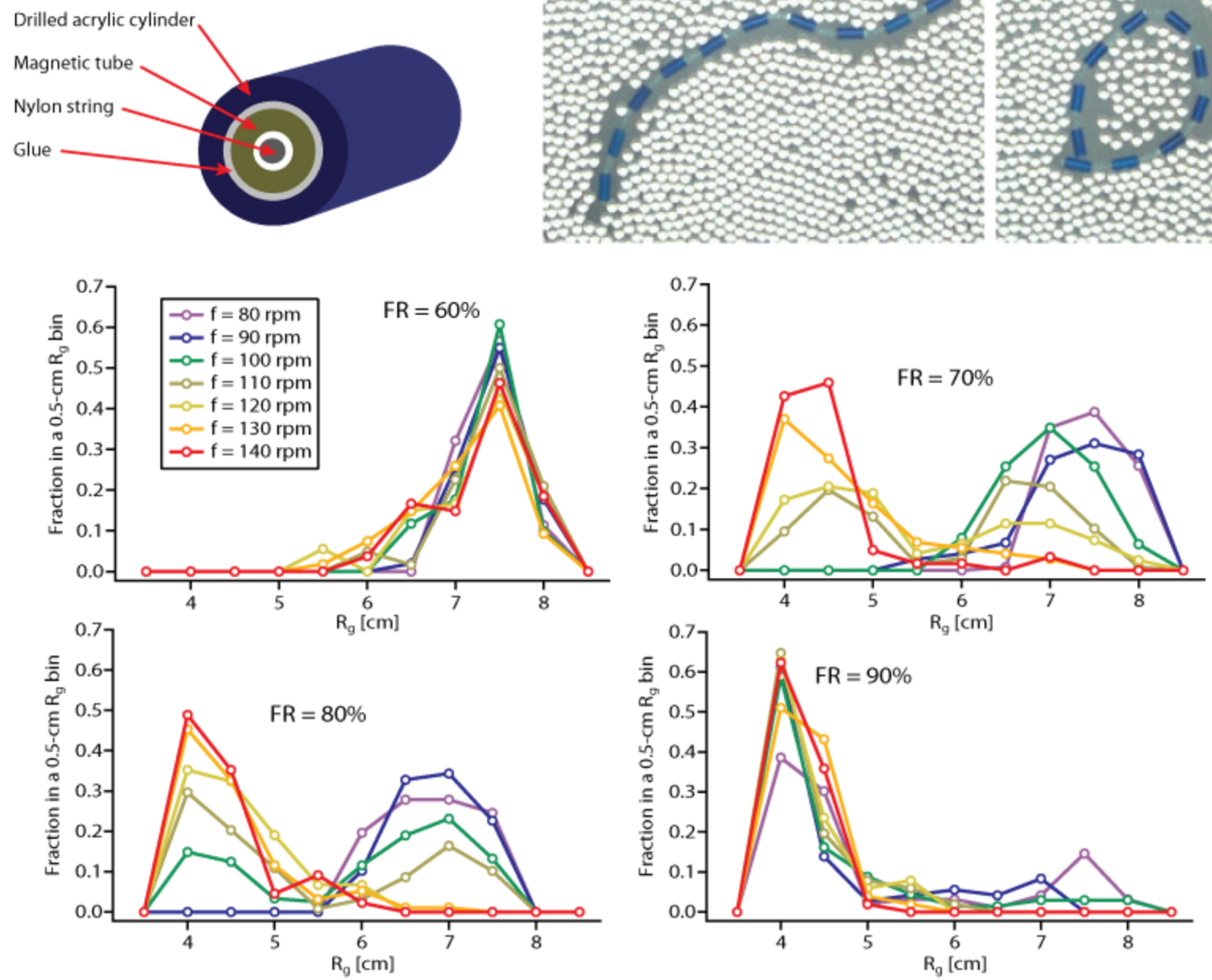

d

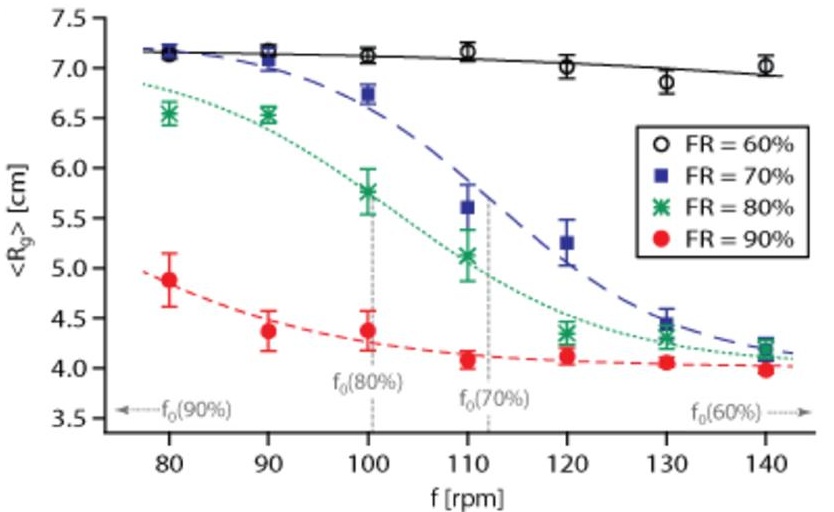

e

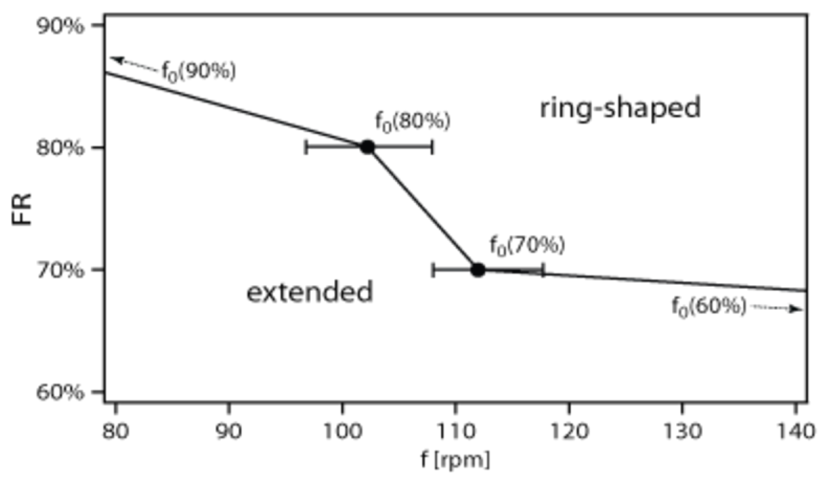

